\title{
Index episode-of-care propensity-matched comparison of transforaminal lumbar interbody fusion (TLIF) techniques: open traditional TLIF versus midline lumbar interbody fusion (MIDLIF) versus robot-assisted MIDLIF
}

\author{
Mikhail Lew P. Ver, MD, Jeffrey L. Gum, MD, Charles H. Crawford III, MD, Mladen Djurasovic, MD, \\ R. Kirk Owens II, MD, Morgan Brown, MS, Portia Steele, APRN, and Leah Y. Carreon, MD, MSc \\ Norton Leatherman Spine Center, Louisville, Kentucky
}

OBJECTIVE Posterior fixation with interbody cage placement can be accomplished via numerous techniques. In an attempt to expedite recovery by limiting muscle dissection, midline lumbar interbody fusion (MIDLIF) has been described. More recently, the authors have developed a robot-assisted MIDLIF (RA-MIDLIF) technique. The purpose of this study was to compare the index episode-of-care (iEOC) parameters between patients undergoing traditional open transforaminal lumbar interbody fusion (tTLIF), MIDLIF, and RA-MIDLIF.

METHODS A retrospective review of a prospective, multisurgeon surgical database was performed. Consecutive patients undergoing 1- or 2-level tTLIF, MIDLIF, or RA-MIDLIF for degenerative lumbar conditions were identified. Patients in each cohort were propensity matched based on age, sex, smoking status, BMI, diagnosis, American Society of Anesthesiologists (ASA) class, and number of levels fused. Index EOC parameters such as length of stay (LOS), estimated blood loss (EBL), operating room (OR) time, and actual, direct hospital costs for the index surgical visit were analyzed.

RESULTS Of 281 and 249 patients undergoing tTLIF and MIDLIF, respectively, 52 cases in each cohort were successfully propensity matched to the authors' first 55 RA-MIDLIF cases. Consistent with propensity matching, there was no significant difference in age, sex, BMI, diagnosis, ASA class, or levels fused. Spondylolisthesis was the most common indication for surgery in all cohorts. The mean total iEOC was similar across all cohorts. Patients undergoing RA-MIDLIF had a shorter average LOS (1.53 days) than those undergoing either MIDLIF (2.71 days) or TTLIF (3.58 days). Both MIDLIF and RA-MIDLIF were associated with lower EBL and less OR time compared with TTLIF.

CONCLUSIONS Despite concerns for additional cost and time while introducing navigation or robotic technology, a propensity-matched comparison of the authors' first 52 RA-MIDLIF surgeries with tTLIF and MIDLIF showed promising results for reducing OR time, EBL, and LOS without increasing cost.

https://thejns.org/doi/abs/10.3171/2019.9.SPINE1932

KEYWORDS episode of care; cost analysis; cost of care; transforaminal lumbar interbody fusion; TLIF; midline interbody fusion; robot-assisted MIDLIF; surgical technique

$\mathrm{V}$ ARIOUs techniques are used for posterior fixation of degenerative lumbar disorders. Midline lumbar interbody fusion (MIDLIF), using the cortical bone trajectory (CBT) screw insertion introduced by Santoni et al., is a minimally invasive alternative to the traditional pedicle screw insertion used in conjunction with transforaminal lumbar interbody fusion (TLIF) of the lumbar spine. . $23,29,32$ There are several advantages of
CBT and MIDLIF reported in literature. Soft-tissue dissection is limited to the region of the pars interarticularis, and retraction is minimal while still allowing a corridor for decompression and grafting for posterior spinal fusion. ${ }^{23,26}$ The CBT reduces possible injury to the neurovascular structures while achieving higher insertional torque and pullout strength compared with the traditional pedicle screw trajectory. ${ }^{18-20,26}$ Recent evidence has shown

ABBREVIATIONS ASA = American Society of Anesthesiologists; $C B T=$ cortical bone trajectory; $E B L=$ estimated blood loss; iEOC = index episode of care; $L O S=$ length of stay; MIDLIF = midline lumbar interbody fusion; $O R=$ operating room; RA = robot-assisted; TLIF = transforaminal lumbar interbody fusion; tTLIF = traditional open TLIF. SUBMITTED January 8, 2019. ACCEPTED September 16, 2019.

INCLUDE WHEN CITING Published online January 24, 2020; DOI: 10.3171/2019.9.SPINE1932. 
its utility in degenerative lumbar disorders where clinical measures such as postoperative pain, functional and disability scores, and postoperative fusion were similar to the traditional technique with lower postoperative complications. ${ }^{2,11,26}$

The introduction of robot-assisted (RA) spinal instrumentation further advanced the benefits of increased pedicle screw placement accuracy and reduction of pedicle violation. Various studies and meta-analyses have reported similar, if not better, accuracy with RA pedicle screw placement. ${ }^{3}$ The push for minimally invasive surgery and increasing demand for accuracy of spinal instrumentation has driven the increasing number of RA spine surgeries performed worldwide. We recently developed an RA-CBT technique in an effort to minimize tissue dissection even further.

It has been established that index episode-of-care (iEOC) parameters, such as length of stay (LOS), estimated blood loss (EBL), and cost, for minimally invasive spine procedures are reduced compared with those of the traditional open procedures..$^{25,27,34,37,38}$ However, iEOC analysis specific to newer technologies such as those utilized in navigated MIDLIF and RA-MIDLIF are lacking. We hypothesized that actual and direct costs and LOS in RA-MIDLIF are similar when compared with MIDLIF and perhaps even lower than in traditional open lumbar procedures such as TLIF. The purpose of this study was to determine differences in iEOC between RA-MIDLIF, MIDLIF, and a traditional open TLIF (tTLIF) procedure for degenerative lumbar disorders.

\section{Methods}

\section{Study Design and Parameters}

This is a single-center, retrospective cohort study approved by the University of Louisville institutional review board. We identified patients from our surgical database who had 1- or 2-level posterior fixation with posterior interbody placement (tTLIF or MIDLIF), performed by 4 different fellowship-trained surgeons for degenerative lumbar conditions. These patients were compared with our first 55 patients who had RA-MIDLIF for similar conditions. The RA-MIDLIF procedure was performed by 1 surgeon (J.L.G.), who also had patients in the other 2 cohorts. Patients with nondegenerative causes were excluded from the study. Baseline data included age, sex, BMI, diagnosis, American Society of Anesthesiologists (ASA) Physical Classification System class, and iEOC parameters including 1) EBL, 2) operating room (OR) time, 3) LOS, and 4) variable direct costs. The cost analysis was performed from the hospital perspective. Thus, cost was the actual direct costs incurred by the hospital and not an estimated amount such as Medicare-allowable rates or charges. OR time was calculated from incision to skin closure time, and LOS was calculated by Centers for Medicare and Medicaid Services guidelines of midnights in the hospital.

\section{Surgical Techniques}

Traditional Open TLIF

A midline skin incision was carried out posteriorly, fol- lowed by a longitudinal incision to the fascia. Subperiosteally, the paravertebral muscles were dissected free from the spinous process, lamina, facets, and the transverse processes. Lateral radiographs were obtained to confirm the level. Pedicle screws were inserted via the freehand technique bilaterally. Decompression was carried out sequentially via laminectomy, facetectomy, annulotomy, and discectomy, typically unilaterally. Endplates were prepared and followed by placement of an interbody device with bone graft. Based on surgeon preference, graft material was pre- and/or postpacked with regard to the interbody cage. Rod insertion to complete posterior instrumentation was performed, with bone grafting posteriorly in the facet joints and in the posterolateral gutters. Graft and biological selection were based on surgeon preference and not standardized.

\section{Navigated MIDLIF}

A smaller posterior midline skin incision was made, followed by a longitudinal incision to the fascia. The paravertebral muscles were elevated from the spinous process to expose the pars. Navigation via $\mathrm{O}$-arm and StealthStation system imaging (Medtronic) was used to assist with instrumentation. The reference frame/array was attached to the vertebra above the planned upper instrumented vertebra in all cases. The starting point of the cortical screw was identified medial to the lateral edge of the pars at the inferior border of the transverse process. Cortical tracks were prepared with a typical trajectory at $20^{\circ}$ medial to lateral and $30^{\circ}-45^{\circ}$ caudal to cephalad followed by lineto-line tapping. Decompression was performed through laminectomy, facetectomy, anulotomy, and discectomy, followed by endplate preparation and interbody device placement with bone graft. Cortical screws were inserted, the rod construct was completed, and bone grafting (surgeon preference) was done posterior to the contralateral facet.

\section{RA-MIDLIF}

Protocol-specific preoperative CT scanning (1-mm slices) was performed in all cases. Prior to surgery, the instrumentation was planned using robotic software (Mazor $\mathrm{X}$, Mazor Robotics, Ltd.). Screw trajectories were manipulated to converge on the skin in line with the disc, which allows for a smaller incision compared with that used for navigated MIDLIF. Exposure similar to that of MIDLIF was performed, followed by bilateral facetectomies. A Schanz pin was inserted into the right posterior superior iliac spine, and the robotic arm (Mazor X) was connected. Oblique and anteroposterior fluoroscopy images were used to register the patient's anatomy to the preoperative $\mathrm{CT}$ scan. The robotic arm was then used to guide the cortical tracks and drilled with a $3.0-\mathrm{mm}$ drill bit. Contralateral screws were inserted after line-to-line tapping, leaving the ipsilateral screws out of the way for decompression. Decompression and interbody fusion were carried out as described in the MIDLIF and tTLIF procedures. Preplanned cortical screws were inserted, the rod construct was completed, and bone grafting (surgeon preference) was done posteriorly at the contralateral facet. 
TABLE 1. Patient demographics for each propensity-matched cohort for tTLIF, MIDLIF, and RA-MIDLIF

\begin{tabular}{|c|c|c|c|c|}
\hline & tTLIF & MIDLIF & RA-MIDLIF & p Value \\
\hline Age, yrs, mean (SD) & $53.08(12.21)$ & $54.44(12.92)$ & $54.46(12.95)$ & 0.816 \\
\hline $\mathrm{BMI}, \mathrm{kg} / \mathrm{m}^{2}$, mean (SD) & $32.15(8.26)$ & $31.54(6.36)$ & $30.60(6.78)$ & 0.545 \\
\hline \multicolumn{4}{|l|}{ Sex } & \multirow{3}{*}{0.199} \\
\hline Males & 13 & 21 & 20 & \\
\hline Females & 39 & 31 & 32 & \\
\hline \multicolumn{4}{|l|}{ No. of fusion levels } & \multirow{3}{*}{0.017} \\
\hline 1 & 35 & 46 & 35 & \\
\hline 2 & 17 & 6 & 17 & \\
\hline \multicolumn{4}{|l|}{ Diagnosis } & \multirow{4}{*}{0.881} \\
\hline Stenosis & 7 & 8 & 7 & \\
\hline Spondylolisthesis & 29 & 31 & 27 & \\
\hline Mechanical disc collapse & 16 & 13 & 18 & \\
\hline \multicolumn{4}{|l|}{ ASA class } & \multirow{4}{*}{0.575} \\
\hline 1 & 0 & 1 & 0 & \\
\hline$\|$ & 20 & 18 & 23 & \\
\hline III & 13 & 21 & 20 & \\
\hline
\end{tabular}

\section{Statistical Analysis}

All statistical analyses were performed using IBM SPSS for Macintosh (version 25, IBM Corp.). As there may be some selection bias as to the type of surgery performed, tTLIF and MIDLIF patients were each propensity matched to our initial cohort of RA-MIDLIF patients using age, sex, BMI, available ASA class, number of levels fused, and diagnosis to provide meaningful comparisons between the surgical groups. The propensity scoring technique is a logistic regression technique that matches multiple characteristics to produce balanced or similar groups for comparison..$^{1,7,17}$ This allows multiple matching of characteristics without the need for one-on-one matching between selected cases and controls. ${ }^{1,30}$ Differences between the groups based on the defined iEOC outcomes were analyzed using one-way ANOVA with the statistical significance set at $\mathrm{p} \leq 0.01$. Post hoc analysis with Bonferroni correction ( $p$ value $\leq 0.01$ was considered significant) was done to identify the cohort that was different from the other cohorts.

\section{Results}

We identified 218 patients undergoing tTLIF and 249 undergoing MIDLIF procedures. We successfully matched 52 cases from each surgical cohort to our first
55 RA-MIDLIF cases (Table 1). The majority of patients in each cohort were female and had an average BMI > 30 $\mathrm{kg} / \mathrm{m}^{2}$. The most common diagnosis for all 3 groups was spondylolisthesis with an ASA class III. Baseline demographic data were compared and consistent with propensity matching; there were no statistically significant differences among the 3 groups based on age, sex, BMI, ASA class, and number of levels fused (Table 1).

The outcomes for iEOC parameters are reported in Table 2 . The tTLIF cohort had the greatest EBL (mean \pm SD: $434.13 \pm 328.25 \mathrm{ml})$ compared with either the MIDLIF cohort $(300.48 \pm 256.94 \mathrm{ml})$ or the RA-MIDLIF cohort $(226.35 \pm 210.80 \mathrm{ml}, \mathrm{p}=0.001)$. Similarly, OR time was also longest in the tTLIF cohort (233.65 \pm 78.96 minutes) compared with either the RA-MIDLIF cohort (188.71 \pm 42.78 minutes) or the MIDLIF cohort (189.57 \pm 42.81 minutes, $p<0.001)$. LOS was statistically significantly different among the 3 cohorts, with the RA-MIDLIF cohort having the shortest $\operatorname{LOS}(1.53 \pm 0.81$ days), followed by the MIDLIF cohort $(2.71 \pm 1.49$ days $)$ and the tTLIF cohort $(3.58 \pm 1.65$ days, $\mathrm{p}<0.001)$. The total variable direct costs were similar between the tTLIF $(\$ 18,204)$ and RAMIDLIF $(\$ 18,277)$ cohorts, with the MIDLIF procedure costing less at $\$ 16,545$, although the difference was not statistically significant $(\mathrm{p}=0.140)($ Table 2$)$.

TABLE 2. Index iEOC parameters and cost analysis for tTLIF, MIDLIF, and RA-MIDLIF

\begin{tabular}{|c|c|c|c|c|}
\hline & \multicolumn{3}{|c|}{ Mean (SD) } & \multirow[b]{2}{*}{ p Value } \\
\hline & tTLIF & MIDLIF & RA-MIDLIF & \\
\hline $\mathrm{EBL}, \mathrm{ml}$ & $434.13(328.25)$ & $300.48(256.94)$ & $226.35(210.80)$ & 0.001 \\
\hline OR time, mins & $233.65(78.96)$ & 189.57 (42.81) & $188.71(42.78)$ & $<0.001$ \\
\hline LOS, days & $3.58(1.65)$ & $2.71(1.49)$ & $1.53(0.81)$ & $<0.001$ \\
\hline Variable direct cost & $\$ 18,204.42(6335.47)$ & $\$ 16,544.94(3027.19)$ & $\$ 18,276.57(4630.67)$ & 0.140 \\
\hline
\end{tabular}




\section{Discussion}

The MIDLIF technique is a less-invasive procedure utilizing a CBT screw trajectory while providing an open corridor with direct visualization for posterior decompression and spinal fusion through a posterior midline approach. Since its initial description by Santoni et al. in 2009, there have been several modifications for CBT screw insertion that have facilitated the technique of application by using a new starting point at the isthmus, fluoroscopic guidance, and CT-guided navigation. 2,26,32 Using the CBT technique allowed Mizuno et al. to utilize MIDLIF as another alternative to an open lumbar spinal fusion. ${ }^{23}$

There are several reported advantages with MIDLIF using a cortical bone screw trajectory, including 1) maximal contact of the screw with a higher-density bony region and increased final insertional torque (ideal for an osteoporotic spine); ${ }^{16,20}$ 2) medial starting point for the screw on the pars interarticularis, which translates to a smaller dissection, less blood loss, and lower postoperative pain; ${ }^{26,32}$ 3) midline exposure adequate for decompression, interbody fusion, and posterior bone grafting, ${ }^{23}$ 4) safer screw trajectory traversing laterally and cephalad away from the dural sac, nerve roots, and anterior vascular structures; ${ }^{23}$ and 5) lower risk of injuring the medial branch nerves, which is associated with postoperative radiculitis after traditional pedicle screw insertion. ${ }^{21}$ Indications for MIDLIF are similar to those for traditional lumbar fusion techniques such as tTLIF or posterior lumbar interbody fusion, but the MIDLIF has advantages in obese patients since it requires less exposure for instrumentation and in osteopenic patients who would benefit from better screw fixation and increased pullout strength. ${ }^{16,18,20}$ Several studies, including a meta-analysis, comparing CBT screw fixation with traditional pedicle screws in lumbar interbody fusions have shown no difference in outcomes of fusion, pain, functional, and disability scores while having lower postoperative complications in CBT fixation. . $^{2,11,14,26,31}$

The smaller incision in RA-MIDLIF compared with that in navigated MIDLIF and the shorter surgical time lead to better recovery kinetics. The smaller incision, less muscle dissection, and the shorter operative time lead to fewer physiological insults to the patient, resulting in a shorter LOS.

There has been much development with RA spine surgery in the recent decade. Published meta-analyses on comparative studies have shown the superiority of RA instrumentation over the freehand technique on outcomes of fusion and postoperative functional status, although there are no available long-term studies. ${ }^{3,4,8,24,40}$ There are several individual trials that have shown better accuracy in screw placement, lower radiation exposure, shorter OR time, and shorter average LOS with RA instrumentation compared with procedures using freehand screw insertion. $5,10,12,13,15$ With establishment of the perioperative benefits of RA instrumentation, its application to our MIDLIF procedure adds further benefit by reducing unnecessary soft-tissue dissection and retraction while at the same time increasing the accuracy of CBT screw insertion. ${ }^{12,15,33}$ As described in Methods, our navigated MIDLIF technique utilizes a spinous process clamp on the $U I V+1$, requiring a more cranial dissection than that necessary for screw placement alone. Utilizing the robotic planning software on preoperative CT scanning can decrease the size of the MIDLIF incision. We typically draw a line at the level of the disc(s) out to the skin and then manipulate the cortical screw trajectories to converge on the skin. This technique allows the surgeon to truly minimize the incision and exposure.

Cost analysis for RA spine surgery is lacking, more so with the RA-MIDLIF procedure, as - to our knowledgewe are the first to present the technique and any associated analysis. ${ }^{11,35}$ Based on the established benefits of RA surgery and the increasing need for data on parameters of iEOC costs, we have explored the different drivers of cost with our first 52 RA-MIDLIF cases compared with a propensity-matched cohort of navigated MIDLIF and tTLIF cases.5,40 For 1- or 2-level degenerative lumbar disorders, the mean EBL and OR time were significantly lower in the two MIDLIF cohorts compared with the tTLIF cohort, and the LOS was shortest in the RA-MIDLIF cohort followed by the MIDLIF cohort and longest in the tTLIF cohort (Fig. 1). These parameters have been shown to influence costs in varying models of healthcare economics. $28,35,36,39$

Cost of care is another parameter on which iEOC analyses are based. Cost of care is usually divided into direct and indirect costs. ${ }^{28}$ Direct costs consist of the actual cost related to patient care, which involves fees attributed to hospital admissions, surgical fees, and implant costs. Meanwhile, indirect costs are related to the value of societal productivity loss resulting from the disease. Our study did not include occupation or time off of work, and we performed our cost analysis from a hospital perspective (actual cost, not Medicare-allowable rates); therefore, we did not include indirect costs. Surgical fees and the cost of implant and supplies are major parameters considered in iEOC analysis for surgical procedures. ${ }^{28,36}$ There were no differences in direct cost for RA-MIDLIF on parameters relating to variable direct costs (Table 2), although it should be considered that the MIDLIF procedure had the lowest supply cost when compared with RA-MIDLIF and tTLIF. This is likely due to the additional disposable supplies required when robot assistance is used; even though navigation carries a disposable cost, it is typically lower than that for the robotic setup. Sharing the granular disposable costs and implant costs is not possible, as these are protected under vendor agreements and vary from institution to institution. Also, although the implant and biological selection was not standardized across all 3 cohorts, it is worth mentioning that our institution utilizes a capitated pricing setup, which typically equalizes cost among different vendors. Use of RA spinal instrumentation does not increase direct overall cost compared with a minimally invasive MIDLIF procedure and an open tTLIF. The comparable costs of enabling technologies such as navigation and RA procedures to traditional lumbar fusion techniques can help allow decision-making from a stakeholder and patient perspective in pursuing RA spinal instrumentation or for decision-making on the purchase of a robot by hospital administrators. 9,35 The breakdown of individual cost components from the iEOC may assist in creating cost-beneficial strategies that will benefit both 


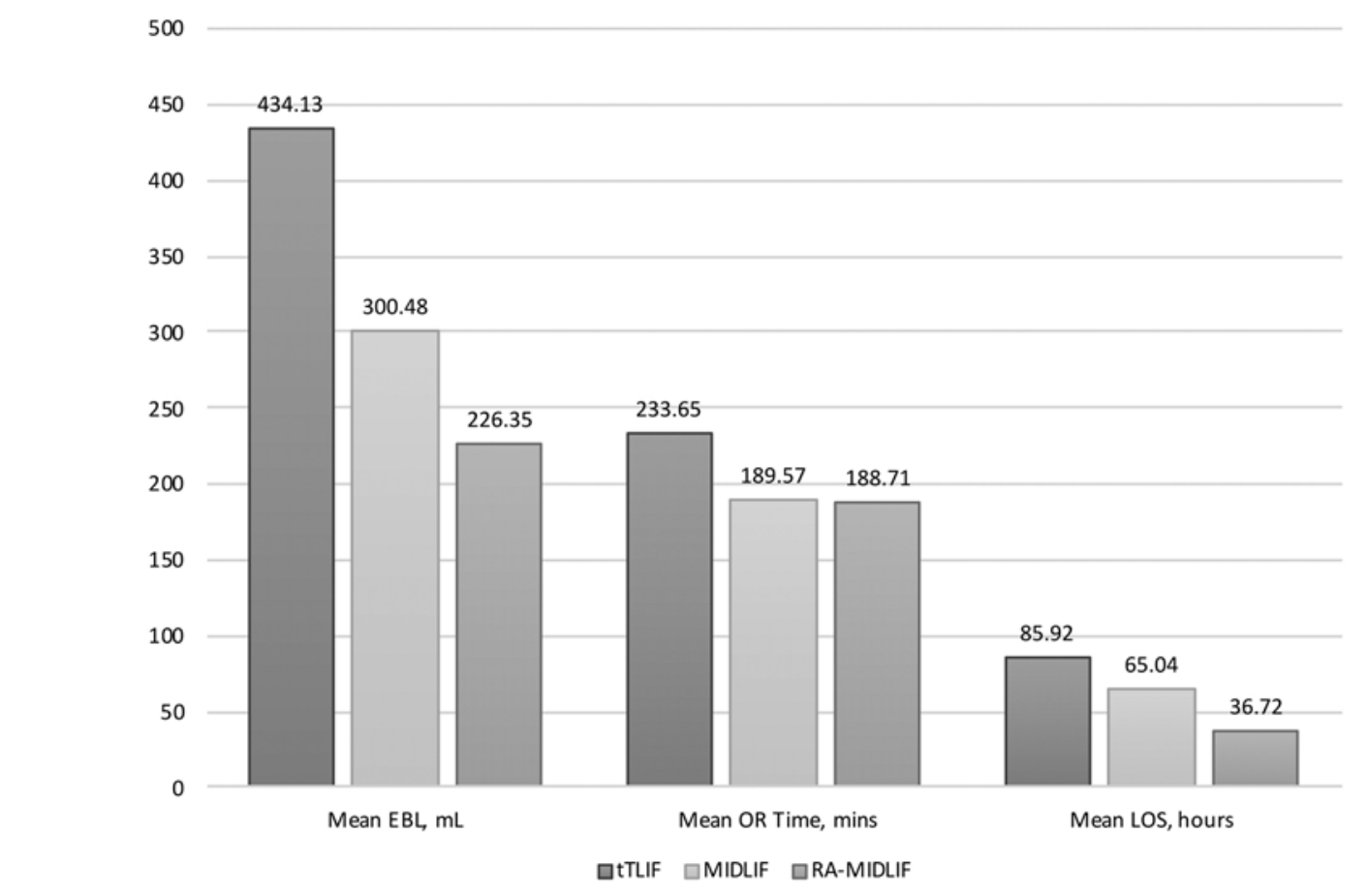

FIG. 1. Significant differences $(p<0.01)$ in iEOC parameters for mean EBL in milliliters, mean OR time in minutes, and LOS in hours.

hospital and patients. This may also guide further research into cost-effectiveness analysis once long-term outcome analyses become available. ${ }^{22}$

\section{Study Limitations}

The main limitation of our study is the limited sample size available for analysis. Costs outside the initial surgical visit were not included, hence limiting the cost analysis to only the iEOC. Individual comorbidities beyond ASA class and patient-reported outcomes were not included in the analysis, all of which may have varying effects on healthcare cost and expenditure per patient. Once longterm follow-up is available, an outcome analysis may provide further information in determining the cost-effectiveness of the RA-MIDLIF and the economic impact of RA spinal surgery on healthcare. Another limitation includes surgeon bias between cohorts. All 4 surgeons contributed to MIDLIF and tTLIF cohorts, but only 1 surgeon performed the RA-MIDLIF procedures.

\section{Conclusions}

Both navigated and RA-MIDLIF cohorts showed promising results in reducing $\mathrm{iEOC}$ parameters with reduction in EBL, OR time, and LOS, and individual parameters for direct costs in a propensity-matched comparison. The introduction of enabling technologies under our study parameters has improved the iEOC profile while maintaining cost. The cost-analysis data provided can be used for a preliminary study to expand into cost-effective analysis and to improve overall healthcare economics for RA spinal instrumentation.

\section{References}

1. D’Agostino RB Jr: Propensity score methods for bias reduction in the comparison of a treatment to a non-randomized control group. Stat Med 17:2265-2281, 1998

2. Delgado-Fernandez J, García-Pallero MÁ, Blasco G, PulidoRivas P, Sola RG: Review of cortical bone trajectory: evidence of a new technique. Asian Spine J 11:817-831, 2017

3. Fan Y, Du JP, Liu JJ, Zhang JN, Qiao HH, Liu SC, et al: Accuracy of pedicle screw placement comparing robot-assisted technology and the free-hand with fluoroscopy-guided method in spine surgery: an updated meta-analysis. Medicine (Baltimore) 97:e10970, 2018

4. Gao S, Lv Z, Fang H: Robot-assisted and conventional freehand pedicle screw placement: a systematic review and meta-analysis of randomized controlled trials. Eur Spine J 27:921-930, 2018

5. Hyun SJ, Kim KJ, Jahng TA, Kim HJ: Minimally invasive robotic versus open fluoroscopic-guided spinal instrumented fusions: a randomized controlled trial. Spine (Phila Pa 1976) 42:353-358, 2017

6. Iwatsuki K, Yoshimine T, Ohnishi Y, Ninomiya K, Ohkawa $\mathrm{T}$ : Isthmus-guided cortical bone trajectory for pedicle screw insertion. Orthop Surg 6:244-248, 2014

7. Joffe MM, Rosenbaum PR: Invited commentary: propensity scores. Am J Epidemiol 150:327-333, 1999

8. Joseph JR, Smith BW, Liu X, Park P: Current applications of robotics in spine surgery: a systematic review of the literature. Neurosurg Focus 42(5):E2, 2017

9. Kahn EN, Ellimoottil C, Dupree JM, Park P, Ryan AM: Variation in payments for spine surgery episodes of care: implications for episode-based bundled payment. J Neurosurg Spine 29:214-219, 2018

10. Kantelhardt SR, Martinez R, Baerwinkel S, Burger R, Giese A, Rohde V: Perioperative course and accuracy of screw positioning in conventional, open robotic-guided and percu- 
taneous robotic-guided, pedicle screw placement. Eur Spine J 20:860-868, 2011

11. Keorochana G, Pairuchvej S, Trathitephun W, Arirachakaran A, Predeeprompan P, Kongtharvonskul J: Comparative outcomes of cortical screw trajectory fixation and pedicle screw fixation in lumbar spinal fusion: systematic review and metaanalysis. World Neurosurg 102:340-349, 2017

12. Keric N, Eum DJ, Afghanyar F, Rachwal-Czyzewicz I, Renovanz M, Conrad J, et al: Evaluation of surgical strategy of conventional vs. percutaneous robot-assisted spinal transpedicular instrumentation in spondylodiscitis. J Robot Surg 11:17-25, 2017

13. Kim HJ, Kang KT, Park SC, Kwon OH, Son J, Chang BS, et al: Biomechanical advantages of robot-assisted pedicle screw fixation in posterior lumbar interbody fusion compared with freehand technique in a prospective randomized controlled trial-perspective for patient-specific finite element analysis. Spine J 17:671-680, 2017

14. Lee GW, Ahn MW: Comparative study of cortical bone trajectory-pedicle screw (cortical screw) versus conventional pedicle screw in single-level posterior lumbar interbody fusion: a 2 -year post hoc analysis from prospectively randomized data. World Neurosurg 109:e194-e202, 2018

15. Lonjon N, Chan-Seng E, Costalat V, Bonnafoux B, Vassal M, Boetto J: Robot-assisted spine surgery: feasibility study through a prospective case-matched analysis. Eur Spine J 25:947-955, 2016

16. Mai HT, Mitchell SM, Hashmi SZ, Jenkins TJ, Patel AA, Hsu WK: Differences in bone mineral density of fixation points between lumbar cortical and traditional pedicle screws. Spine J 16:835-841, 2016

17. Månsson R, Joffe MM, Sun W, Hennessy S: On the estimation and use of propensity scores in case-control and casecohort studies. Am J Epidemiol 166:332-339, 2007

18. Matsukawa K, Taguchi E, Yato Y, Imabayashi H, Hosogane $\mathrm{N}$, Asazuma T, et al: Evaluation of the fixation strength of pedicle screws using cortical bone trajectory: What is the ideal trajectory for optimal fixation? Spine (Phila Pa 1976) 40:E873-E878, 2015

19. Matsukawa K, Yato Y, Imabayashi H, Hosogane N, Asazuma T, Chiba K: Biomechanical evaluation of lumbar pedicle screws in spondylolytic vertebrae: comparison of fixation strength between the traditional trajectory and a cortical bone trajectory. J Neurosurg Spine 24:910-915, 2016

20. Matsukawa K, Yato Y, Imabayashi H, Hosogane N, Asazuma T, Nemoto K: Biomechanical evaluation of the fixation strength of lumbar pedicle screws using cortical bone trajectory: a finite element study. J Neurosurg Spine 23:471-478, 2015

21. Matsukawa K, Yato Y, Nemoto O, Imabayashi H, Asazuma T, Nemoto K: Morphometric measurement of cortical bone trajectory for lumbar pedicle screw insertion using computed tomography. J Spinal Disord Tech 26:E248-E253, 2013

22. McGirt MJ, Parker SL, Asher AL, Norvell D, Sherry N, Devin CJ: Role of prospective registries in defining the value and effectiveness of spine care. Spine (Phila Pa 1976) 39 (22 Suppl 1):S117-S128, 2014

23. Mizuno M, Kuraishi K, Umeda Y, Sano T, Tsuji M, Suzuki $\mathrm{H}$ : Midline lumbar fusion with cortical bone trajectory screw. Neurol Med Chir (Tokyo) 54:716-721, 2014

24. Park SM, Kim HJ, Lee SY, Chang BS, Lee CK, Yeom JS: Radiographic and clinical outcomes of robot-assisted posterior pedicle screw fixation: two-year results from a randomized controlled trial. Yonsei Med J 59:438-444, 2018

25. Parker SL, Mendenhall SK, Shau DN, Zuckerman SL, Godil SS, Cheng JS, et al: Minimally invasive versus open transforaminal lumbar interbody fusion for degenerative spondylolisthesis: comparative effectiveness and cost-utility analysis. World Neurosurg 82:230-238, 2014
26. Phan K, Hogan J, Maharaj M, Mobbs RJ: Cortical bone trajectory for lumbar pedicle screw placement: a review of published reports. Orthop Surg 7:213-221, 2015

27. Phan K, Hogan JA, Mobbs RJ: Cost-utility of minimally invasive versus open transforaminal lumbar interbody fusion: systematic review and economic evaluation. Eur Spine J 24:2503-2513, 2015

28. Rihn JA, Currier BL, Phillips FM, Glassman SD, Albert TJ: Defining the value of spine care. J Am Acad Orthop Surg 21:419-426, 2013

29. Rodriguez A, Neal MT, Liu A, Somasundaram A, Hsu W, Branch CL Jr: Novel placement of cortical bone trajectory screws in previously instrumented pedicles for adjacentsegment lumbar disease using CT image-guided navigation. Neurosurg Focus 36(3):E9, 2014

30. Rosenbaum PR: Model-based direct adjustment. J Am Stat Assoc 82:387-394, 1987

31. Sakaura H, Miwa T, Yamashita T, Kuroda Y, Ohwada T: Cortical bone trajectory screw fixation versus traditional pedicle screw fixation for 2-level posterior lumbar interbody fusion: comparison of surgical outcomes for 2-level degenerative lumbar spondylolisthesis. J Neurosurg Spine 28:57-62, 2018

32. Santoni BG, Hynes RA, McGilvray KC, Rodriguez-Canessa G, Lyons AS, Henson MAW, et al: Cortical bone trajectory for lumbar pedicle screws. Spine J 9:366-373, 2009

33. Schatlo B, Molliqaj G, Cuvinciuc V, Kotowski M, Schaller $\mathrm{K}$, Tessitore E: Safety and accuracy of robot-assisted versus fluoroscopy-guided pedicle screw insertion for degenerative diseases of the lumbar spine: a matched cohort comparison. $\mathbf{J}$ Neurosurg Spine 20:636-643, 2014

34. Singh K, Nandyala SV, Marquez-Lara A, Fineberg SJ, Oglesby M, Pelton MA, et al: A perioperative cost analysis comparing single-level minimally invasive and open transforaminal lumbar interbody fusion. Spine J 14:1694-1701, 2014

35. Smith HE, Rihn JA, Brodke DS, Guyer R, Coric D, Lonner $\mathrm{B}$, et al: Spine care: evaluation of the efficacy and cost of emerging technology. Am J Med Qual 24 (6 Suppl):25S31S, 2009

36. Ugiliweneza B, Kong M, Nosova K, Huang KT, Babu R, Lad $\mathrm{SP}$, et al: Spinal surgery: variations in health care costs and implications for episode-based bundled payments. Spine 39:1235-1242, 2014

37. Vertuani S, Nilsson J, Borgman B, Buseghin G, Leonard C, Assietti R, et al: A cost-effectiveness analysis of minimally invasive versus open surgery techniques for lumbar spinal fusion in Italy and the United Kingdom. Value Health 18:810-816, 2015

38. Wang MY, Lerner J, Lesko J, McGirt MJ: Acute hospital costs after minimally invasive versus open lumbar interbody fusion: data from a US national database with 6106 patients. J Spinal Disord Tech 25:324-328, 2012

39. Yeramaneni S, Ames CP, Bess S, Burton D, Smith JS, Glassman S, et al: Center variation in episode-of-care costs for adult spinal deformity surgery: results from a prospective, multicenter database. Spine J 18:1829-1836, 2018

40. Yu L, Chen X, Margalit A, Peng H, Qiu G, Qian W: Robotassisted vs freehand pedicle screw fixation in spine surgerya systematic review and a meta-analysis of comparative studies. Int J Med Robot 14:e1892, 2018

\section{Disclosures}

Dr. Gum: employee of Norton Healthcare; consultant for Medtronic, DePuy, Acuity, K2M, PacMed, and NuVasive; direct stock ownership in Cingulate Therapeutic; clinical or research support for this study from Intellirod, Integra, Pfizer, and International Spine Study; and royalties from Acuity. Dr. Crawford: 
consultant for Alphatec, DePuy Synthes, Medtronic, and NuVasive. Dr. Djurasovic: consultant for Medtronic and NuVasive. Dr. Owens: consultant for Medtronic and NuVasive; and support of non-study-related clinical or research effort from Pfizer, Intellirod, and Texas Scottish Rite Hospital. Dr. Brown: employee of Norton Healthcare. Dr. Carreon: employee of Norton Healthcare; consultant for AOSpine; funds for travel from the University of Southern Denmark and University of Louisville; institutional research funds from OREF, NIH, ISSG, SRS, TSRH, Pfizer, and Cerapedics; member of the editorial advisory board for Spine Deformity, The Spine Journal, and Spine; member of University of Louisville IRB; and research committee of SRS.

\section{Author Contributions}

Conception and design: Gum, Crawford, Djurasovic, Owens, Steele, Carreon. Acquisition of data: Crawford, Djurasovic, Owens, Brown, Steele. Analysis and interpretation of data: Ver, Gum, Brown, Carreon. Drafting the article: Ver. Critically revising the article: Ver, Gum. Reviewed submitted version of manuscript: Ver, Gum, Carreon. Approved the final version of the manuscript on behalf of all authors: Ver. Statistical analysis: Brown, Carreon. Administrative/technical/material support: Gum, Crawford, Djurasovic, Owens, Steele, Carreon. Study supervision: Carreon.

\section{Correspondence}

Mikhail Lew P. Ver: Norton Leatherman Spine Center, Louisville, KY.miko.ver@gmail.com. 